\title{
Pterional or Subfrontal Access for Proximal Vascular Control in Anterior Interhemispheric Approach for Ruptured Pericallosal Artery Aneurysms at Risk of Premature Rupture
}

\author{
Jaechan Park, M.D., Ph.D. \\ Department of Neurosurgery, Research Center for Neurosurgical Robotic Systems, Kyungpook National University, Daegu, Korea
}

Objective : Cases of a ruptured pericallosal artery aneurysm with a high risk of intraoperative premature rupture and technical difficulties for proximal vascular control require a technique for the early and safe establishment of proximal vascular control.

Methods : A combined pterional or subfrontal approach exposes the bilateral A1 segments or the origin of the ipsilateral A2 segment of the anterior cerebral artery (ACA) for proximal vascular control. Proximal control far from the ruptured aneurysm facilitates tentative clipping of the rupture point of the aneurysm without a catastrophic premature rupture. The proximal control is then switched to the pericallosal artery just proximal to the aneurysm and its intermittent clipping facilitates complete aneurysm dissection and neck clipping.

Results : Three such cases are reported : a ruptured pericallosal artery aneurysm with a contained leak of the contrast from the proximal side of the aneurysm, a low-lying ruptured pericallosal artery aneurysm with irregularities on its proximal wall, and a multilobulated ruptured pericallosal artery aneurysm with the parasagittal bridging veins hindering surgical access to the proximal parent artery. In each case, the proposed combined pterional-interhemispheric or subfrontal-interhemispheric approach was successfully performed to establish proximal vascular control far from the ruptured aneurysm and facilitated aneurysm clipping via the interhemispheric approach.

Conclusion : When using an anterior interhemispheric approach for a ruptured pericallosal artery aneurysm with a high risk of premature rupture, a pterional or subfrontal approach can be combined to establish early proximal vascular control at the bilateral $\mathrm{A} 1$ segments or the origin of the $\mathrm{A} 2$ segment.

Key Words : Clipping · Interhemispheric approach · Intracranial aneurysms · Proximal vascular control · Pterional approach.

\section{INTRODUCTION}

An anterior interhemispheric approach is mandatory for most pericallosal artery aneurysms $\mathrm{s}^{1,2,5,-9,12,15,17)}$. Using retraction on the medial surface of the frontal lobe, the dissection is carried down into the interhemispheric fissure. The parent artery distal to the aneurysm is generally reached first and followed by careful dissection past the aneurysm to the proximal parent artery. Thus, cases of a ruptured pericallosal artery aneurysm are an exception to the classical vascular principle of

- Received : October 10, 2016 -Accepted : December 15, 2016

- Address for reprints : Jaechan Park, M.D., Ph.D.

Department of Neurosurgery, Kyungpook National University Hospital, 130 Dongdeok-ro, Jung-gu, Daegu 41944, Korea

Tel : +82-53-200-5647, Fax : +82-53-423-0504, E-mail : jparkmd@hotmail.com

This is an Open Access article distributed under the terms of the Creative Commons Attribution Non-Commercial License (http://creativecommons.org/licenses/by-nc/4.0) which permits unrestricted non-commercial use, distribution, and reproduction in any medium, provided the original work is properly cited. 
early achievement of proximal vascular control.

If uncontrolled intraoperative bleeding occurs from a ruptured pericallosal artery aneurysm, a tamponade and suction technique is the only available option, since proximal vascular control can be hampered due to adhesion of the cingulate gyri, peri-aneurysmal adhesion, or blocked operative access to the proximal parent artery by the bleeding rupture point ${ }^{7,18)}$. This situation can lead to damage of the aneurysmal neck and parent artery.

Thus, a safer way to achieve proximal vascular control is needed for cases of a ruptured pericallosal artery aneurysm with a high risk of intraoperative premature rupture and technical difficulties for proximal vascular control. Accordingly, a pterional or subfrontal approach was combined to expose the bilateral A1 segments or the origin of the ipsilateral A2 segment of the ACA, allowing early and safe establishment of proximal vascular control far from the rupture point. While somewhat laborious, this combined approach is effective in avoiding catastrophic premature bleeding for high-risk patients.

A combined pterional-interhemispheric approach has already been reported in literature, yet has been limited to cases of multiple aneurysms requiring both surgical approaches ${ }^{3,6)}$. In contrast, the proposed combined approach is intended to establish safe and early proximal vascular control for ruptured pericallosal artery aneurysms located in the interhemispheric fissure. The technical details and indications for the proposed combined approach are described using 3 surgical cases.

\section{SURGICAL TECHNIQUE}

The patient is positioned supine and the head placed in a neutral position. For aneurysms that lie inferior to the genu of the corpus callosum, the neck is then extended approximately 15 degrees, whereas the neck is not extended for aneurysms lying close to the genu of the corpus callosum.

A combined pterional-paramedian craniotomy is created for the pterional approach to achieve proximal vascular control (Fig. 1A), while a frontal craniotomy bordered by the lateral orbital roof and a superior sagittal sinus is created for the combined subfrontal-interhemispheric approach (Fig. 1B).

For the combined subfrontal-interhemispheric approach, a bicoronal scalp incision is made and reflected anteriorly using an interfascial dissection of the temporalis muscle, as originally described by Yasargil et al. ${ }^{20)}$. Meantime, for the combined pterional-interhemispheric approach, the temporalis muscle is separated and elevated with the scalp ipsilaterally, while an interfascial dissection of the temporalis muscle is performed contralaterally.

To create a cranial bone flap combining a pterional craniotomy and frontal paramedian craniotomy, 5 burrholes are made as follows : 1) the first hole is a keyhole just superior to the frontozygomatic suture, 2) the second hole is placed inferiorly on the temporal squamous bone, 3 ) the third hole is placed at the posterior aspect of the exposure just inferior to the superior temporal line, and 4) the fourth and fifth holes are placed on each side of the midline at the posterior extent of the paramedian craniotomy part. To avoid dimples in the forehead, no burrholes are made either side of the midline at the anterior extent of the craniotomy. The anteromedial bor-
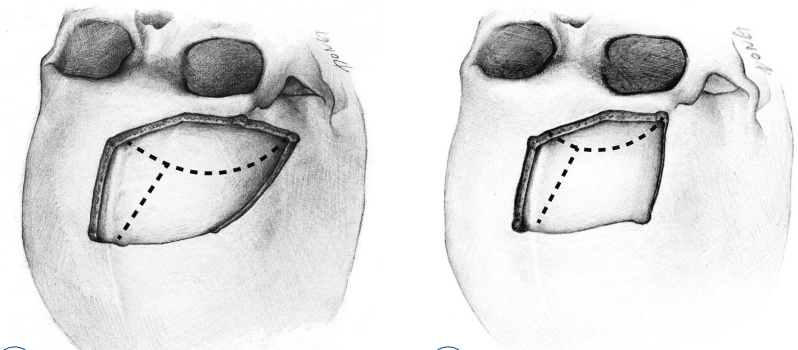

(A)

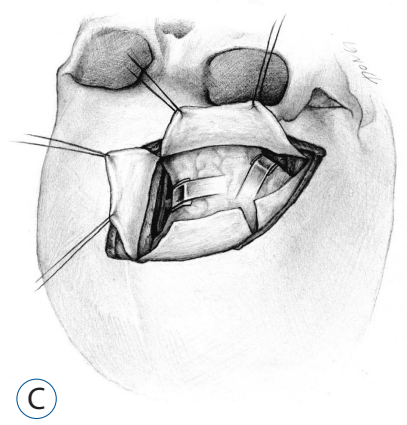

Fig. 1. Illustrations depicting the combination of a pterional or subfrontal approach and interhemispheric approach. A : Combined pterionalparamedian craniotomy for a pterional-interhemispheric approach. The dural opening (dotted line) creates a C-shaped dural flap based anteriorly and another dural flap based medially along the superior sagittal sinus. B : Frontal craniotomy bordered by the lateral orbital roof and a superior sagittal sinus for a combined subfrontal-interhemispheric approach. C : Alternation between a pterional approach and anterior interhemispheric approach. 
der of the craniotomy is performed diagonally to avoid the frontal sinus. To create a free bone flap, the anterior and posterior extent of the craniotomy covering the superior sagittal sinus are cut using a narrow drill bit without a foot plate, while the other burrholes are connected using a narrow drill bit with a protective foot plate. Drilling the sphenoid ridge and frontal floor prominences completes the combined pterionalparamedian craniotomy.

The dural opening is initially performed in a C-shaped fashion, with the dural flap based anteriorly. Another dural incision is then connected to the C-shaped dural incision with the dural flap based medially along the superior sagittal sinus. In cases of severe brain swelling, an intraoperative ventriculostomy is performed using Paine's technique or its modifica$\operatorname{tion}^{14,16)}$.

A self-retaining retractor is first inserted inferior to the frontal cortex using a pterional or subfrontal approach. A carotid cistern, proximal sylvian fissure, and chiasmatic cistern are opened to expose the bilateral Al segments of the ACA. Furthermore, gyrus rectus resection and entrance into the interhemispheric fissure can expose the origin of the ipsilateral A2 segment. The retractor is then moved and inserted medial to the frontal cortex (Fig. 1C). When the inferior edge of the falx and the cingulate gyri adjacent to the ruptured aneurysm are reached, the retractor is re-inserted inferior to the frontal cortex to apply temporary clips to the origin of the ipsilateral A2 segment or bilateral A1 segments. While the origin of the ipsilateral A2 segment is the most optimal and safest point far from the ruptured pericallosal artery aneurysm, bilateral A1 segments can also be used for proximal vascular control. The ipsilateral A1 segment is clipped intermittently, following continuous clipping of the contralateral Al segment. The duration of the temporary clipping should be minimized to avoid compromising the bilateral ACA territories.

Finally, the aneurysm is dissected and a tentative clip is applied to the rupture point. The proximal vascular control is then switched to the pericallosal artery just proximal to the aneurysm, followed by complete aneurysm dissection and neck clipping.

\section{Case 1 : Ruptured pericallosal artery aneurysm with contained leak}

A 75-year-old woman presented with sudden mental deterioration, and a computed tomography (CT) scan showed a subarachnoid hemorrhage (SAH) in the basal cisterns and anterior interhemispheric fissure. Subsequent computed tomography angiography (CTA) showed an aneurysm (16 mm diameter) at the junction of the right pericallosal and callosomarginal arteries, along with a contained leak of the contrast from the aneurysm (Fig. 2A, B). As the contained
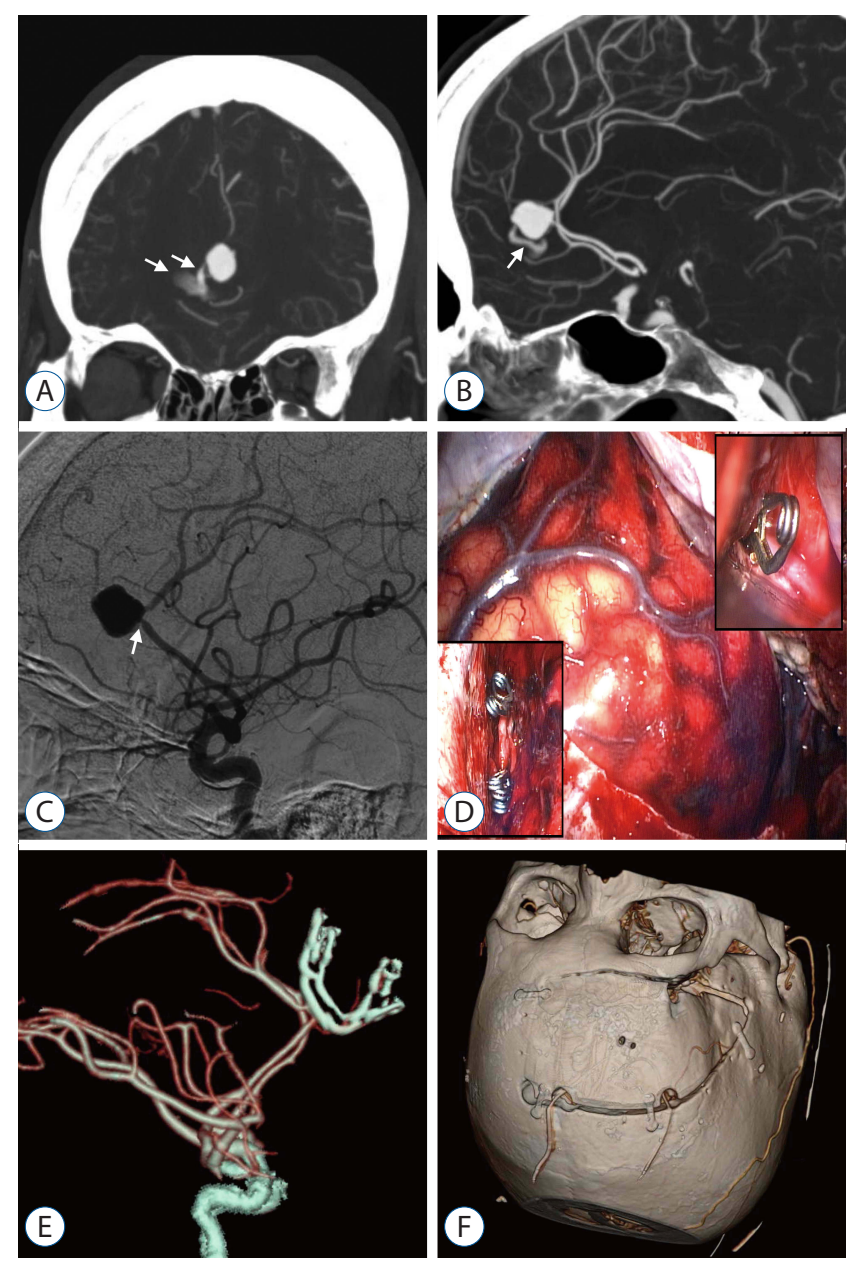

Fig. 2. Case 1. A : Coronal image of the CTA revealing contrast extravasation (arrows) from a ruptured pericallosal artery aneurysm. B : Sagittal image of the CTA revealing contrast extravasation (arrow) from the ruptured pericallosal artery aneurysm. C : DSA revealing severe stenosis (arrow) of the parent artery and a wide neck of the pericallosal artery aneurysm. D : Intraoperative photographs of the combined pterional-interhemispheric approach. Note the left insert, showing the clipped pericallosal artery aneurysm via the anterior interhemispheric approach, and the right insert, showing the temporary clip applied to the origin of the ipsilateral A2 segment via the pterional approach. E : Postoperative CTA demonstrating complete aneurysmal clipping without compromise of the parent artery. $\mathrm{F}$ : Postoperative CT of the head showing the combined pterional-paramedian craniotomy. CTA : computed tomography angiography, DSA : digital subtraction angiography. 
leak was located proximal to the aneurysm, the use of an anterior interhemispheric approach to establish proximal vascular control would inevitably have resulted in a premature rupture. In addition, digital subtraction angiography (DSA) revealed severe stenosis of the parent artery and a wide-neck aneurysm, both of which are poor indications for endovascular coiling (Fig. 2C).

Thus, a combined pterional-paramedian craniotomy on the right side allowed a combined pterional-interhemispheric approach (Fig. 2D). After an intraoperative ventriculostomy using a modified Paine's technique for acute hydrocephalus, proximal vascular control was achieved at the origin of the ipsilateral A2 segment via a pterional approach. Using retraction medial to the frontal cortex interhemispherically, the aneurysm was accessed and a tentative clip was applied to the rupture point. Intermittent proximal vascular control at the pericallosal artery just proximal to the aneurysm and complete aneurysm dissection allowed permanent aneurysm neck clipping without a premature rupture.

The postoperative recovery was uneventful. The postoperative CTA showed complete clipping without any residual aneurysm or parent artery compromise (Fig. 2E). A reconstructed CT shows the combined pterional-paramedian craniotomy (Fig. 2F).

\section{Case 2 : Low-lying pericallosal artery aneurysm}

A 63-year-old woman presented with a sudden severe headache related to an SAH. CTA and DSA showed a ruptured aneurysm (10 $\mathrm{mm}$ diameter) at the junction of the left pericallosal and callosomarginal arteries (Fig. 3A). The aneurysm resided inferior to the genu of the corpus callosum with the neck as low as $14 \mathrm{~mm}$ from the level of the planum sphenoidale (Fig. 3B). In addition, the proximal side of the aneurysm exhibited irregularities with the possibility of a rupture point. Thus, surgical access to the proximal parent artery through a narrow corridor in close proximity to the ruptured aneurysm represented a high risk of premature aneurysm rupture.

A rectangular frontal craniotomy was created with an anterolateral border close to the lateral orbital roof and medial border exposing the superior sagittal sinus (Fig. 3C, D). The bilateral A1 segments were then used for proximal vascular control. After applying a temporary clip to the contralateral A1 segment via a subfrontal approach (Fig. 3E), interhemispheric access to the pericallosal artery aneurysm was at-
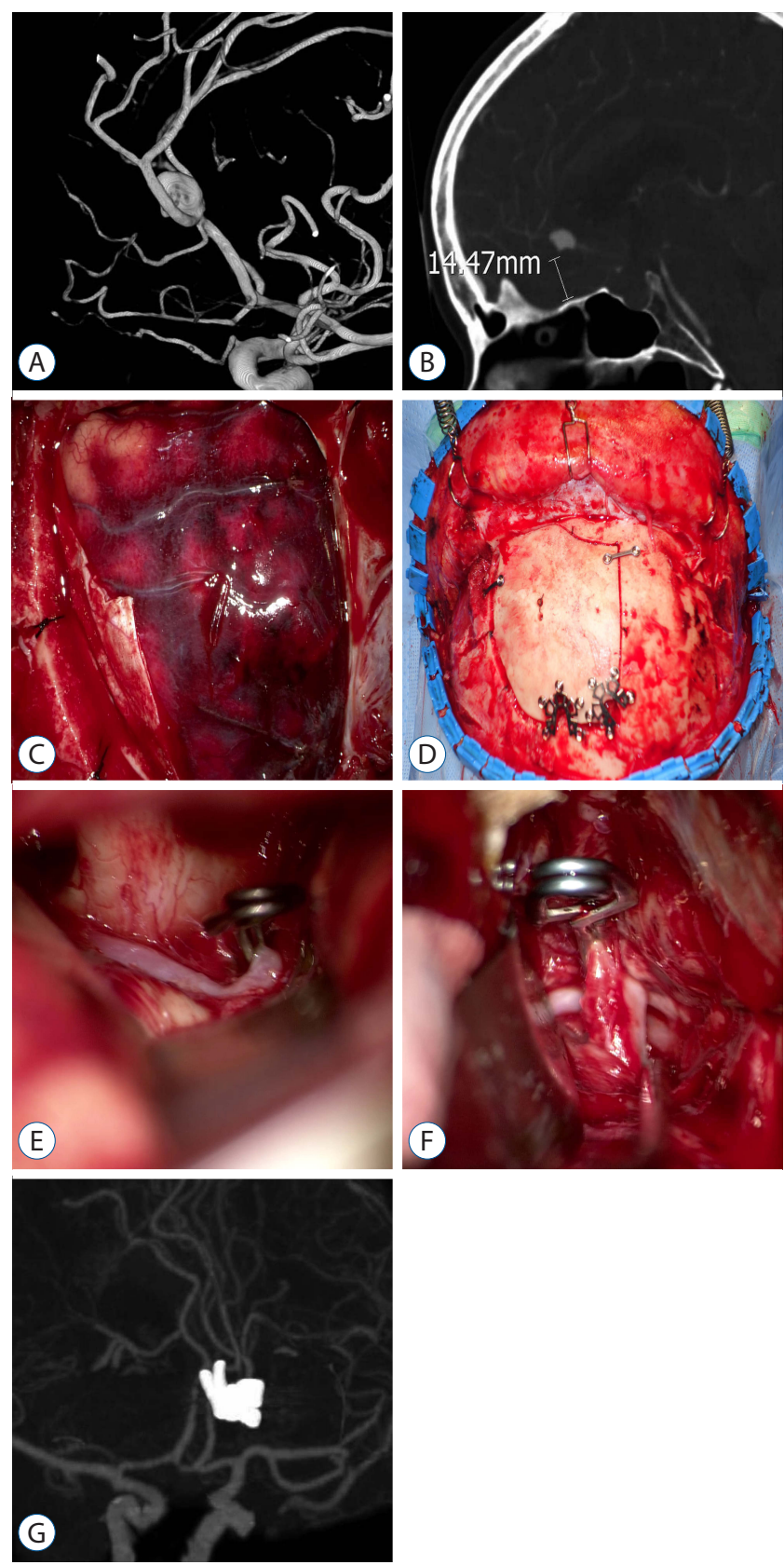

Fig. 3. Case 2. A : DSA showing a ruptured pericallosal artery aneurysm arising from the origin of the callosomarginal artery. B : Sagittal image of the CTA revealing a low-lying pericallosal artery aneurysm as low as $14 \mathrm{~mm}$ from the level of the planum sphenoidale. $C$ : Intraoperative photograph showing the frontal craniotomy for the subfrontal-interhemispheric approach. D : Intraoperative photograph showing the cranial bone flap fixed in place after clipping the aneurysm. The craniotomy is bordered by the lateral orbital roof and a superior sagittal sinus. E: Intraoperative photograph showing the bilateral A1 segments via the subfrontal approach. A temporary clip is applied to the contralateral A1 segment. F : Intraoperative photograph showing the pericallosal artery aneurysm clipping via the interhemispheric approach. G : Postoperative CTA demonstrating complete aneurysmal clipping without compromise of the parent artery. DSA : digital subtraction angiography, CTA : computed tomography angiography. 
tempted using a neuronavigation system. When the interhemispheric dissection was in close proximity to the aneurysm, a second temporary clip was applied to the ipsilateral A1 segment via the subfrontal approach. The proximal vascular control softened the aneurysm, facilitated dissection, and helped tentative clipping of the rupture point, thereby allowing the proximal clips to be removed from the bilateral A1 segments. Plus, intermittent proximal control just proximal to the aneurysm facilitated complete aneurysm dissection and neck clipping (Fig. 3F).

The postoperative recovery was uneventful and the patient returned to normal life. A postoperative CTA showed successful obliteration of the aneurysm and preservation of the parent artery (Fig. 3G).

\section{Case 3 : Multilobulated ruptured pericallosal ar- tery aneurysm with parasagittal bridging veins hindering access to proximal artery}

A 58-year-old woman presented with an SAH in the basal cisterns and anterior interhemispheric fissure. DSA showed a multilobulated pericallosal artery aneurysm arising from the origin of the callosomarginal artery and a small saccular aneurysm in the left M1 segment of the middle cerebral artery (MCA). The extraordinary multilobulated appearance of the pericallosal artery aneurysm raised concerns of an intraoperative rupture (Fig. 4A). Moreover, pterional or subfrontal access to the proximal artery was required as surgical access to the proximal parent artery via an anterior interhemispheric approach was hindered by frontal parasagittal bridging veins (Fig. 4B). Besides, a concomitant MCA aneurysm favored a combined pterional approach.

A combined pterional-paramedian craniotomy was created on the left side and an intraoperative ventriculostomy was performed to achieve brain relaxation. After applying a temporary clip to the contralateral A1 segment via the pterional approach (Fig. 4C), a self-retaining retractor was inserted interhemispherically posterior to the frontal parasagittal bridging veins (Fig. 4D). When the pericallosal artery was reached distally, it was followed anteriorly to open the interhemispheric fissure from inside-out. Notwithstanding, the aneurysm ruptured prematurely. Immediate clipping of the ipsilateral A1 segment via the pterional approach dramatically reduced the aneurysmal bleeding and facilitated dissection and tentative clipping of the aneurysm. Switching to intermittent prox-
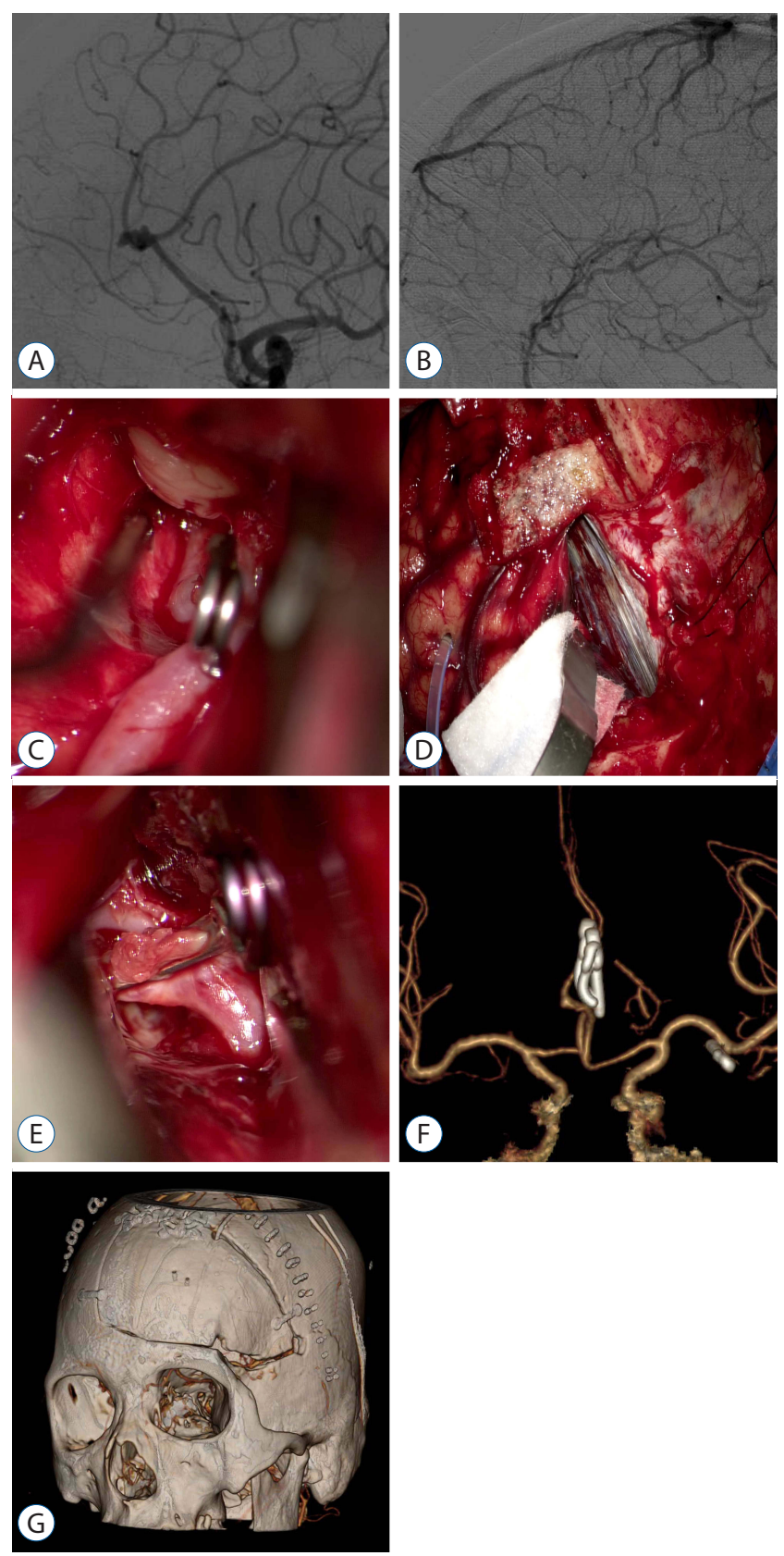

Fig. 4. Case 3. A : DSA showing a left pericallosal artery aneurysm arising from the origin of the callosomarginal artery. $B$ : Venous phase of the left carotid angiogram showing parasagittal bridging veins hindering surgical access to the proximal parent artery via an interhemispheric approach. C : Intraoperative photograph showing bilateral A1 segments via a pterional approach. A temporary clip is applied to the contralateral A1 segment. D : Intraoperative photograph showing the anterior interhemispheric approach. E : Intraoperative photograph showing aneurysm clipping via the anterior interhemispheric approach. F : Postoperative CTA demonstrating complete clipping of the pericallosal artery aneurysm and MCA aneurysm. G : Postoperative CT of the head showing the combined pterional-paramedian craniotomy. DSA : digital subtraction angiography, CTA : computed tomography angiography, MCA : middle cerebral artery. 
imal control just proximal to the aneurysm allowed permanent neck clipping of the aneurysm without any ischemic complications (Fig. 4E). Finally, the small MCA aneurysm was also dissected and clipped via the pterional approach.

The postoperative recovery was uneventful and the postoperative CTA showed successful obliteration of the aneurysms (Fig. 4F). A reconstructed CT shows the combined pterionalparamedian craniotomy (Fig. 4G).

\section{DISCUSSION}

Proximal vascular control using a pterional or subfrontal approach is recommended in cases of a high risk of premature rupture related to surgical access and dissection of a pericallosal artery aneurysm and its parent artery. Angiographic findings, such as a contained leak of the contrast, multilobulated appearance, and large-sized ruptured aneurysms, can all be considered as risk factors of intraoperative rupture during peri-aneurysmal dissection. Furthermore, the proposed surgical technique can be mandatory if proximal vascular control is predicted to be difficult. For example, low-lying pericallosal artery aneurysms, parasagittal bridging veins hindering surgical access to the parent artery, and a rupture point or contained leak in the proximal side of the aneurysm are all indicative of difficulties in accessing the proximal parent artery interhemispherically.

The proposed combined approach also requires certain technical precautions to minimize the occlusion time of the parent vessel. The proximal vascular occlusion at the distant site should be delayed until the peri-aneurysmal area is reached interhemispherically, applied intermittently based on frequent shifting between the pterional/subfrontal and interhemispheric approaches, and only used until tentative clipping of the rupture point, thereby allowing intermittent proximal vascular control in proximity to the aneurysm interhemispherically. In addition, effective methods of cerebral protection, including induced hypertension and burst suppression on electroencephalography using propofol or pentobarbital, also need to be used.

The endovascular option is temporary balloon occlusion of a proximal vessel, although previous related reports have mostly been limited to aneurysms arising from large vessels, such as the paraclinoid internal carotid artery and basilar ar- tery ${ }^{4,10,11,13,19)}$. The critical problem with using an endovascular procedure as an adjunct to microsurgical clipping is the risk of a thromboembolism. Plus, intravenous heparinization to prevent a distal embolism can produce a bleeding tendency, making the arachnoid dissection more difficult and even generating an intracranial hemorrhage.

The proposed combined approach has certain limitations. First, it requires a larger craniotomy than a simple interhemispheric approach. Second, frequent shifting between the pterional and interhemispheric approach to alternate the proximal vascular control and aneurysmal dissection is a cumbersome process for less experienced surgeons. Third, if one or two parasagittal bridging veins are sacrificed in the anterior interhemispheric approach, the circulatory load of the venous system is collateralized via adjacent venous drainage, such as the frontal ascending draining veins and superficial sylvian vein. Thus, additional compromise of the superficial sylvian vein with the pterional approach can lead to venous infarction. Fortunately, the dissection of the proximal sylvian fissure for access to the A1 or proximal A2 segment is only minimal.

\section{CONCLUSION}

When using an anterior interhemispheric approach for a ruptured pericallosal artery aneurysm, if the risk of intraoperative rupture is high and proximal vascular control is technically difficult, a combined pterional or subfrontal approach to expose the bilateral A1 segments or the origin of the ipsilateral A2 segment of the ACA can be useful for proximal vascular control.

\section{References}

1. de Sousa AA, Dantas FL, de Cardoso GT, Costa BS : Distal anterior cerebral artery aneurysms. Surg Neurol 52 : 128-135; discussion 135-136, 1999

2. Ducruet AF, Connolly ES Jr : Microsurgery of distal anterior cerebral artery aneurysms in Winn HR (ed) : Youmans Neurological Surgery, ed 6. Elsevier Saunders : Philadelphia, 2011, pp3853-3861

3. Edner $G$ : One-session operation via bilateral craniotomies for multiple aneurysms after subarachnoid haemorrhage. Br J Neurosurg 5 : 55 60, 1991

4. Elhammady MS, Nakaji P, Farhat H, Morcos JJ, Aziz-Sultan MA : Balloon- 
assisted clipping of a large paraclinoidal aneurysm : a salvage procedure. Neurosurgery 65 : E1210-E1211; discussion E1211, 2009

5. Kawashima M, Matsushima T, Sasaki T : Surgical strategy for distal anterior cerebral artery aneurysms : microsurgical anatomy. J Neurosurg 99 : 517-525, 2003

6. Kiyofuji S, Inoue T, Tamura A, Saito I : Combined interhemispheric and pterional approach for ACOM and left MCA aneurysms. Neurosurg Focus 38(Video Suppl 1) : Video 15, 2015

7. Lawton MT : Seven aneurysms : tenets and techniques for clipping. Thieme Medical Publishers, Inc., New York, 2011, pp 147-163

8. Lee JW, Lee KC, Kim YB, Huh SK : Surgery for distal anterior cerebral artery aneurysms. Surg Neurol 70 : 153-159; discussion 159, 2008

9. Martines F, Blundo C, Chiappetta F : Surgical treatment of the distal anterior cerebral artery aneurysms. J Neurosurg Sci 40 : 189-194, 1996

10. Mizoi K, Takahashi A, Yoshimoto T, Fujiwara S, Koshu K : Combined endovascular and neurosurgical approach for paraclinoid internal carotid artery aneurysms. Neurosurgery $33:$ 986-992, 1993

11. Mizoi K, Yoshimoto T, Takahashi A, Ogawa A : Direct clipping of basilar trunk aneurysms using temporary balloon occlusion. J Neurosurg 80 : 230-236, 1994

12. Navarro R, Chao K, Steinberg GK : Microsurgical management of distal anterior cerebral artery aneurysms : from basic to complex, a video review of four cases. Acta Neurochir (Wien) 155 : 2115-2119, 2013
13. Ng PY, Huddle D, Gunel M, Awad IA : Intraoperative endovascular treatment as an adjunct to microsurgical clipping of paraclinoid aneurysms. J Neurosurg 93 : 554-560, 2000

14. Paine JT, Batjer $H H$, Samson D : Intraoperative ventricular puncture. Neurosurgery 22(6 Pt 1) : 1107-1109, 1988

15. Park J, Hamm IS : Anterior interhemispheric approach for distal anterior cerebral artery aneurysm surgery : preoperative analysis of the venous anatomy can help to avoid venous infarction. Acta Neurochir (Wien) 146 : 973-977; discussion 977, 2004

16. Park J, Hamm IS : Revision of Paine's technique for intraoperative ventricular puncture. Surg Neurol 70 : 503-508; discussion 977, 2008

17. Proust $F$, Toussaint $P$, Hannequin $D$, Rabenenoïna $C$, Le Gars $D$, Fréger $P$ : Outcome in 43 patients with distal anterior cerebral artery aneurysms. Stroke 28 : 2405-2409, 1997

18. Srinivasan J, Getch CC, Chandler JP, Batjer HH : Intraoperative aneurysm rupture in Le Roux PD, Winn HR, Newell DW (eds) : Management of cerebral aneurysms. Saunders, Philadelphia, 2004, pp 867-874

19. Thorell W, Rasmussen P, Perl J, Masaryk T, Mayberg M : Balloon-assisted microvascular clipping of paraclinoid aneurysms. Technical note. J Neurosurg $100:$ 713-716, 2004

20. Yasargil MG, Reichman MV, Kubik S : Preservation of the frontotemporal branch of the facial nerve using the interfascial temporalis flap for pterional craniotomy. Technical article. J Neurosurg 67 : 463-466, 1987 